\title{
Tandem $\alpha$-Alkylation-Asymmetric Transfer Hydrogenation of Acetophenones
}

Metal-Catalyzed

Asymmetric

Synthesis and

Stereoselective

Reactions

\section{Key words}

transfer

hydrogenation

alkylation

ruthenium

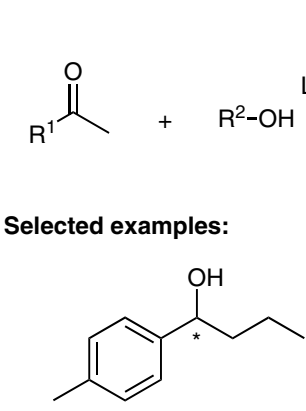

$35 \%$ yield, $84 \%$ ee<smiles>CCCC(O)c1cccc(OC)c1</smiles>

$43 \%$ yield, $86 \%$ ee

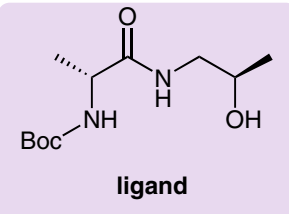<smiles>[3H]C[C@@H]([18OH])CO</smiles>

$$
\text { ligand }(1.1 \mathrm{~mol} \%)
$$

$\mathrm{LiCl}(10 \mathrm{~mol} \%), t$-BuOK $(50 \mathrm{~mol} \%)$

$$
\text { DMSO, } 65-40^{\circ} \mathrm{C}
$$

\section{ligand}

Significance: The authors present the first example of a direct formation of enantiomerically enriched secondary alcohols from ketones and primary alcohols by a tandem $\alpha$-alkylation-asymmetric transfer hydrogenation process using $\left[\mathrm{Ru}\left(\mathrm{p} \text {-cymene) } \mathrm{Cl}_{2}\right]_{2}\right.$ as catalyst in the presence of an amino acid hydroxy amide as ligand.<smiles>CCCC(O)c1ccc(Br)cc1</smiles>

$9 \%$ yield, $79 \%$ ee<smiles>CCCCCC(O)c1ccc(C)cc1</smiles>

$34 \%$ yield, $88 \%$ ee<smiles>CCCCCC(O)c1ccc(OC)cc1</smiles>

$28 \%$ yield, $83 \%$ ee<smiles>CCCCCC(O)c1ccc(C(F)(F)F)cc1</smiles>

$19 \%$ yield, $57 \%$ ee

Proposed mechanism:
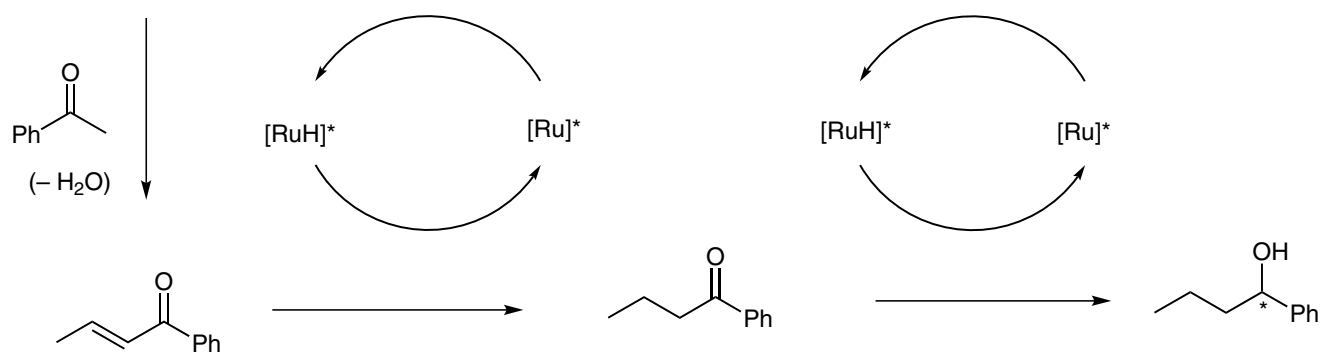

Comment: Diversely substituted acetophenones were successfully converted into chiral secondary alcohols via the borrowing hydrogen methodology in moderate yields and in moderate to good enantiomeric excess. In this process, primary alcohols served as both alkylating and reducing agents. 